\title{
TROIS FOIS PAR MOIS ? DIX FOIS PAR JOUR ? MONTAIGNE ET LA RÉGLEMENTATION DU MARIAGE DANS «SUR DES VERS DE VIRGILE » (ESSAIS, III, 5)
}

\begin{abstract}
Denis Bjaï, Trois fois par mois? dix fois par jour? Montaigne et la réglementation du mariage dans "Sur des vers de Virgile » (Essais, III, 5) [Three times a month ? Ten times a day ? Montaigne and marriage regulations in "Upon some verses of Virgil"], Studia Romanica Posnaniensia, Adam Mickiewicz University Press, Poznań, vol. XXXVIII/1: 2011, pp. 23-34. ISBN 978-83-232-2279-8. ISSN 0137-2475. DOI 10.2478/v10123-011-0003-0.

In his chapter "Upon some lines of Virgil" (Essays, III, 5), Montaigne relates the complaint of a poor Catalonian woman, to whom her husband fulfilled his conjugal duties at least ten times a day, and the arbitrament the Queen of Aragon gave in her favour (?). This spicy story actually raises a tricky point of law, that Nicolas Bohier (Decisiones Aurece, 1544), Antoine du Verdier (Les diverses leçons, 1577) and Guillaume Bouchet (Les Serées, 1584) had previously studied. After Montaigne, the case will be dealt with again in the works of Cholières and Brantôme.
\end{abstract}

Sans tourner inutilement autour du pot, nous emprunterons notre entrée en matière à Rabelais ${ }^{1}$, à ce plaisant dialogue du Cinquième livre entre Panurge et un Frère Fredon qui ne lui répond que par monosyllabes, au moment où ils en viennent à parler des « sœurs en charité », si accommodantes envers les moines :

PANURGE. Que disent-elles en culletant ? FREDON. mot. [...]

PAN. Comment couchez[-vous] ensemble ? FR. nuds.

PAN. Par ledit serment qu'avez faict, quantes fois de bon compte ordinairement le faictes-vous par jour? FR. six.

PAN. Et de nuict? FR. dix.

- Cancre [chancre], dist frere Jehan, le paillard ne daigneroit passer seize, il est honteux [timide $]^{2}$.

Un moine paillard est ici plaisamment dénoncé par un autre, puisque, depuis le Gargantua, on sait frère Jean pourvu d'un beau nez et sans doute d'un autre généreux

${ }^{1}$ Son nom se rencontre une seule fois dans les Essais, quand, dans le chapitre « Des livres », Montaigne le range, avec Boccace et les Basia de Jean Second, parmi les lectures de divertissement « dignes qu'on s'y amuse » (éd. J. Balsamo, M. Magnien et C. Magnien-Simonin, Paris, Gallimard, 2007, p. 430 [texte de 1595] - ce sera notre édition de référence).

${ }^{2}$ Euvres complètes, éd. M. Huchon, Paris, Gallimard, 1994, ch. XXVII, p. 795. 
appendice (ad formam nasi cognoscitur ad te leuaui [À la forme du nez on connaît celle du vers toi j'ai levé]) $)^{3}$. Mais en écho à un chapitre symétrique du Tiers livre ${ }^{4}$, la question a aussi à voir avec le mariage et avec la place consentie, dans ce cadre, à la sexualité, que théologiens et juristes n'ont cessé de définir et de réglementer. Un simple coup d'œil sur l'index des éditions anciennes des Essais, en l'occurrence celui de l'édition L'Angelier de $1602^{5}$, montre tout l'intérêt que Montaigne a porté à son tour au sujet, avec une entrée Mariage qui enregistre, entre autres lieux, « Il est dissous par impuissance », «Il refuse les conditions de l'amour », « Il est semblable aux cages », « Ils [les mariages] ne doivent être pour la beauté seule et désirs d'amour », « Se marier sans s'épouser est trahison », et une entrée Femme plus abondante encore, incluant en particulier « Femme se plaignant de la besogne de son mari, autre le faisant appeler en justice pour contraire effet », «Femme paillarde punie par son mari », «Elles [les femmes] n'ont tort de refuser une trop grande continence », "Elles sont plus ardentes à l'amour que les hommes ». Tous ces points sont abordés dans un seul et même chapitre des Essais, «Sur des vers de Virgile » (III, 5), où Montaigne, derrière ce « titre-façade $»^{6}$, réfléchit librement sur l'amour, le mariage, la sexualité, la jalousie... Allons, là encore, droit au but en partant de la manchette « Femme se plaignant de la besogne de son mari, autre le faisant appeler en justice pour contraire effet ». La seconde d'entre elles est l'épouse du philosophe platonicien Polémon, qui, à en croire Diogène Laërce (IV, 17), traîna son mari devant le juge en arguant « qu'il alloit semant en un champ sterile le fruit deu au champ genital $\aleph^{7}$, métaphore proverbiale pour désigner les pratiques homosexuelles - là où le latin d'Ambrogio Traversari, que Montaigne avait sous les yeux, disait plus crûment « quod adolescentibus congrederetur ». Elle annonce d'autres femmes insatisfaites, comme la jeune épousée négligée par un mari impuissant, au prétexte fallacieux « qu'il avoit faict vingt postes la nuict precedente » (p. 909), ou la reine Jeanne de Naples faisant assassiner André de Hongrie, son premier époux, en quelque sorte pour tromperie sur la marchandise (« sur ce qu'aux courvées matrimoniales, elle ne luy trouvoit ny les parties, ny les efforts, assez respondants à l'esperance qu'elle en avoit conceue, à veoir sa taille, sa beauté, sa jeunesse et disposition », p. 929) ${ }^{8}$, ou encore comme Messaline (évoquée à deux

${ }^{3}$ Éd. citée, ch. XL, p. 112 (incipit des Psaumes).

${ }^{4}$ « Comment frere Jan joyeusement conseille Panurge » (ch. XXVII).

5 Reproduit dans l'éd. J. Céard et al. des Essais parue à la Pochothèque (Paris, LGF, 2001), pp. 1763-1807.

${ }^{6}$ Terme repris à P. Henry, «Les titres façades, la censure et l'écriture défensive chez Montaigne», Bulletin de la Société des Amis de Montaigne, $5^{\text {e }}$ série, n² 24, oct. - déc. 1977, pp. 11-28.

${ }^{7}$ Éd. Balsamo et al., p. 898.

8 Montaigne écarte l'autre explication, de nature politique, avancée par l'historien qu'il démarque ici, Jacques de Lavardin (« poussée aussi de la conspiration de ceux de son sang, aspirant à la souveraineté », Histoire de Scanderbeg, Paris, Guillaume Chaudière, 1576, livre X, f. 384r ${ }^{\circ}$ ). À moins qu'il ne s'inspire plutôt de l'Histoire du royaume de Naples de Collenuccio, traduite par D. Sauvage, et où André est désigné comme Andreasso (plus proche du Andreosse de Montaigne). Cf. Brantôme, Recueil des Dames, I, vII (éd. É. Vaucheret, Paris, Gallimard, 1991, pp. 198-202). 
reprises dans le chapitre), capable d'endurer, en une nuit, pas moins de vingt-cinq assauts $^{9}$ et s'amourachant de C. Silius jusqu'à l'épouser en public, du vivant même de Claude. Mais Montaigne examine aussi le cas inverse, moins attendu semble-t-i ${ }^{10}$, d'une « femme se plaignant de la besogne de son mari ». L'évocation de ce mari intempérant est préparée par celle d'un obscur empereur romain (ou du moins devenu tel pour nous, car c'est à la Renaissance un exemple bien connu de paillardise $)^{11}$. Il s'agit de Proculus, symétrique masculin de Messaline, comme elle, quoiqu'à deux siècles de distance, «maistr[e] ouvri[er] et fameux en ceste besogne » (p. 896) : à en croire l'Histoire Auguste, « luy despucela bien en une nuict dix vierges Sarmates ses captives » (sur les cent qu'il avait faites prisonnières et qu'il se vantait d'avoir toutes déflorées en moins de quinze jours). Ce Proculus Cæsar était déjà allégué dans le Tiers livre, entre Hercule (le séducteur des cinquante filles de Thespios) et Mahomet, parmi les « fabuleux ribaulx » dont Panurge entendait éclipser les exploits sexuels ${ }^{12}$. Mais c'est l'exemple suivant, plus longuement développé par Montaigne, qui retiendra davantage notre attention :

Et que sur le different advenu à Cateloigne, entre une femme, se plaignant des efforts trop assiduelz de son mari (Non tant à mon advis qu'elle en fust incommodee, car je ne crois les miracles qu'en foy, comme pour retrancher soubs ce pretexte, et brider en ce mesme, qui est l'action fondamentale du mariage, l'authorité des maris envers leurs femmes : Et pour montrer que leurs hergnes [leur hargne], et leur malignité passent outre la couche nuptiale, et foulent aux pieds les graces et douceurs mesmes de Venus) à laquelle plainte, le mary respondoit, homme vrayement brutal et desnaturé, qu'aux jours mesme de jeusne il ne s'en sçauroit passer à moins de dix : Intervint ce notable arrest de la Royne d'Aragon : par lequel, apres meure deliberation de conseil, cette bonne Royne, pour donner reigle et exemple à tout temps, de la moderation et modestie requise en un juste mariage : ordonna pour bornes legitimes et necessaires, le nombre de six par jour : Relaschant et quitant beaucoup du besoing et desir de son sexe, pour establir, disoit elle, une forme aysee, et par consequent permanante et immuable. (p. 897)

L'ancien magistrat de la Chambre des Enquêtes du Parlement de Bordeaux se plaît visiblement à rapporter en détail ce cas litigieux et le « notable arrêt » rendue par une femme en faveur d'une autre, pour la protéger, autant que faire se peut, de l'intempérance conjugale. Afin de mettre en lumière le traitement tout à fait original que

${ }^{9}$ Encore Montaigne est-il en retrait par rapport au texte-source de Pline (H.N., X, LXIII, 172) : l'impératrice endura vingt-cinq assauts de plus que la courtisane romaine avec laquelle, cette nuit-là, elle avait rivalisé !

${ }^{10}$ C'est là, comme on le verra, un topos misogyne récurrent dans la littérature du temps. Érasme le place même dans la bouche de la sage Eulalie, quand Xanthippe se plaint à elle de la trop grande ardeur sexuelle de son mari : «Voilà une plainte que l'on n'entend pas communément chez les femmes » (Colloque Uxor mempsigamos sive conjugium, trad. J.-Cl. Margolin, in Éloge de la Folie, Adages [...], éd. Cl. Blum, A. Godin et al., Paris, Robert Laffont, coll. « Bouquins », p. 290).

${ }_{11}$ Voir, entre autres, Petrus Crinitus, De honnesta disciplina, VIII, 7, « De his qui fœecundiores fuerint ad prolem procreandam, ac Proculi Augusti verba de ingenti ejusdem libidine »; Ravisius Textor, Officina, section «Libidinosi et lascivi »; H.C. Agrippa, De incertitudine, ch. 63, « De arte meretricia ».

${ }^{12}$ Ch. XXVII, éd. citée, p. 437 (dans une revue de libidinosi incluant Messaline, p. 436). 
lui réserve Montaigne, nous examinerons les textes dont il a pu s'inspirer ou qu'il a lui-même inspirés, la ligne de démarcation n'étant pas, comme on le verra, toujours aisé à établir : par ordre d'entrée en scène, les Decisiones Burdegalenses de Nicolas Bohier, Les Diverses Leçons d'Antoine du Verdier, Les Serées de Guillaume Bouchet, Les Matinées de Cholières et les Dames galantes de Brantôme.

Nicolas Bohier est un juriste de profession, successivement professeur à l'Université de Bourges, membre du Grand Conseil du Roi et troisième Président au Parlement de Bordeaux. Ses Decisiones Burdegalenses paraissent en 1567 d'après le Dictionnaire de biographie française (un exemplaire à la $\mathrm{BnF}$, un autre à l'Arsenal), en 1551 d'après le Dictionnaire des lettres françaises. XVI esiècle et le Dictionnaire historique des juristes français (un exemplaire à la $\mathrm{BM}$ d'Avignon), mais la première édition, lyonnaise, sous le titre de Decisiones aurece, remonte à 1544, soit cinq ans seulement après la mort du magistrat (deux exemplaires à la Bibliothèque du Congrès à Washington $^{13}$ ), avec des rééditions en 1547, 1559, 1560 et 1566. La décision 317 porte sur la grave question de savoir s'il faut punir le juge, le parlementaire, le conseiller du roi, le chancelier, le greffier qui connaitrait charnellement une femme comparaissant devant lui, ou estant en justice, ou incarcérée ${ }^{14}$. Elle présente toutes les caractéristiques des gloses juridiques de l'époque, telles que les a définies André Tournon à partir, précisément, d'un extrait des Decisiones : « les commentaires se ramifient souvent en considérations diverses, pour exploiter toutes les possibilités offertes par le sujet $\gg^{15}$. Au fil de ses dix-huit pages, distribuées sur deux colonnes, elle mêle en effet aux références juridiques attendues (en particulier au De legibus connubialibus de Tiraqueau) des réminiscences scripturaires ${ }^{16}$ et des citations poétiques : ici, empruntés à Juvénal, ces mêmes vers de la satire VI relatifs à Messaline que citait Montaigne avant d'enchaîner sur l'anecdote catalane. Celle-ci est, chez Bohier, référée à son auteur, ou à ses auteurs : " Hierony. Paul. », c'est-à-dire le canoniste catalan du XVe siècle Hieronymus Paulus, ou Jeroni Pau ${ }^{17}$, qui allègue, au sujet de l'impuissance (de frigidis et maleficiatis, titre d'une célèbre section des Décrétales), le témoignage d'un episcopus Ilerdensis, un évêque de Lerida (Lleida) :

${ }^{13}$ L'un paru chez Antoine Vincent, l'autre chez Michel Parmentier et Jean-François de Gabiano.

${ }^{14}$ Quid de iudice, aut senatore, siue consiliario Regio, vel proeside, aut cancellario, vel commentariense, qui carnaliter mulierem coram eo, vel curia litigantem, aut carceratam, vel aliam ratione iurisdictionis tantum subditam [cognouerit], an puniatur? (Secunda pars aurearum decisionum, Lyon, Antoine Vincent, 1547, f. $341 v^{\circ}$, col. 1).

${ }^{15}$ Montaigne. La glose et l'essai, 1983, $2^{\mathrm{de}}$ édition revue et corrigée, Paris, Champion, 2000, p. 153.

16 Tirés des Proverbes, ch. 30, les versets 15 et 16 sur les trois choses insatiables et la quatrième qui ne dit jamais assez, à savoir l'enfer, le vagin, la terre et le feu (éd. citée, f. $346 v^{\circ}$, col. 1).

${ }^{17}$ Il ne semble toutefois mentionné ici que pour son Prouinciale omnium ecclesiarum cathedralium uniuersi orbis, généralement reproduit à la suite de sa Practica cancellarice apostolica, et qui recense, parmi les évêques suffragants de l'archevêque de Tarragone, celui de Lleida (éd. Venise, E. Reagazola et D. Cavalcalupus, 1572, p. 244). 
ce Léridien a vu de son temps un homme de Catalogne doué d'une telle puissance sexuelle que tous les jours il honorait sa femme trente fois, laquelle s'en plaignit en secret au roi d'Aragon. On fit venir le mari qui reconnut qu'il en était bien ainsi. C'est pourquoi le roi lui défendit, sous peine de la vie, d'honorer sa femme plus de six fois par jour, pour ne pas, dit-il, la mettre en danger de mort. Il ne faut pas tant s'étonner, ici, de la puissance du mari que de la plainte de l'épouse. Même si en effet les femmes osent moins que les hommes [Code de Justinien, livre 9, titre 8, Ad legem Iuliam maiestatis, $5^{\mathrm{e}}$ loi, Quisquis cum militibus...], elles n'en sont pas moins un tant soit peu plus audacieuses dans les choses honteuses et obscènes ainsi que dans l'acte amoureux, comme le glosent ad loc. Cynus [de Pistoia], Albericus [de Rosate] et [Bartholomeus] Salicetus, ainsi que, sur le titre 11 du même livre, De mulieribus [quce se seruis propriis iunxerunt], Lucas de Penna ${ }^{18}$.

C'est bien le même récit que dans les Essais, à deux différences près : trente assauts journaliers, non pas dix ; et un roi d'Aragon, en lieu et place d'une reine. Du moins sous cette version du texte, qui n'est peut-être pas celle que Montaigne avait sous les yeux. Dans les rééditions ultérieures, on lit « qualibet die uxorem suam x. vicibus cognoscentem », puis « reginam Aragoniæ secrete conquesta fuit $»^{19}$, ce qui nous ramène bien au texte des Essais, débarrassé toutefois des références au Code qui encombraient la prose latine de Bohier.

Plus près de Montaigne, et cette fois en français, l'anecdote est reprise par le bibliographe Antoine du Verdier, dont les lectures variées le conduisent (avant même de rédiger, en concurrence avec La Croix du Maine, sa célèbre Bibliothèque) à donner une suite à la Silva de varia lección du polygraphe sévillan Pero Mejía [Pierre Messie], six livres de Diverses leçons parus à Lyon en 1577 et souvent réédités à la suite du recueil original. Au livre IV, entre un chapitre sur l'origine du mal de Naples, c'està-dire, pour les Français, de la syphilis, et un autre consacré à « ceux qui ont esté les plus feconds à engendrer enfans », ainsi qu'à la « lubricité demesuree de l'Empereur Procule $»^{20}, \mathrm{~s}^{\prime}$ intercale un chapitre XxxIII intitulé « De la brutalité et vilainie qui consiste au coit ou acte venerien. Et d'un homme qui habitoit charnellement avec sa femme dix fois par chacun jour, dequoy elle feit plainte, et ce qui en fut ordonné $»^{21}$. Du Verdier y stigmatise l'intempérance conjugale, sinon celle de la femme, dont la lubricité est hors de tout contrôle (comme l'attestent le portrait de Messaline par Juvénal et le même verset des Proverbes que chez Bohier), du moins celle de l'homme, être

${ }^{18}$ Illum Llerdensem [...] vidisse tempore suo hominem quemdam fuisse in Cathalonia tantum in re Venerea potentem qualibet die uxorem suam XXX. vicibus cognoscentem, quce Regi Aragonice secrete conquesta fuit, vocato viro confessus est ita rem se habere. Quare mandauit ei sub pona capitis ne amplius quam sexies in die uxorem suam cognosceret, ne (ut ait) mortis periculum mulier incurreret. Unde de potentia viri non tantum mirari oportet, quantum de querela uxoris. Licet enim minus audeant foemince quam mares, l. quisquis. C. ad legem Iul. maiestatis. tamen in turpibus obscœnisque atque Venereis actibus sunt audaciusculce... (éd. citée, f. $346 \mathrm{v}^{\circ}$, col. 1-2).

19 Lyon, Claude Ravot, 1567, p. 632, col. 1. Cf. P. Villey, Les sources des Essais, t. IV de l'éd. dite Municipale, Bordeaux, 1920, p. 380 (n. sur la p. 87, 1. 24 [du t. III]).

20 Titre démarqué de celui de Petrus Crinitus (voir supra, n. 10).

${ }^{21}$ Lyon, Estienne Michel, 1580, pp. 325-329. 
raisonnable qui doit savoir modérer ses appétits « et non s'enyvrer de son vin [...] ny se jetter sur sa viande comme un pourceau sur ses raves » (p. 328). Et nous retrouvons, explicitement référé aux Decisiones Burdegalenses, le cas « autant prodigieux comme il est peu croyable » du mari catalan, que Du Verdier transpose consciencieusement en français, dans la version révisée des rééditions (« dix fois par chacun jour », «à la Royne d'Aragon », ibid.). Si le traducteur reprend à son compte l'analyse de Bohier sur ce qui, en l'espèce, doit véritablement provoquer l'étonnement, en ajoutant même aux références juridiques l'argument misogyne selon lequel « les femmes se plaignent souvent du peu, mais non pas du trop » (ibid.), il s'en écarte ensuite pour mettre le comportement de l'époux moins au compte de la puissance sexuelle que de la pure et simple brutalité, c'est-à-dire bestialité, ce qui lui permet de rattacher le cas au thème-titre du chapitre. Mais il ne résiste pas à la tentation d'une digression, abruptement refermée, sur les plantes aphrodisiaques dont aurait pu user le mari, en particulier cette herbe mystérieuse qui, au témoignage de Théophraste repris par Pline et par Athénée ${ }^{22}$, aurait permis à un Indien de copuler en une seule nuit pas moins de soixante-dix fois (p. 329).

Peu avant que Montaigne ne publie le livre III des Essais, à la suite des deux premiers revus et augmentés, deux recueils narratifs « bigarrés », comme l'époque les appréciait, avaient encore rapporté, au tournant des années 1580, le cas extraordinaire du couple catalan : Les Serées du libraire-imprimeur poitevin Guillaume Bouchet et Les Matinées du mystérieux seigneur de Cholières (peut-être le nom de plume de l'homme de loi bourguignon Jean Dagoneau ${ }^{23}$ ). Les trois livres des Serées (ou « soirées » en poitevin) rapportent des entretiens et joyeux devis tenus au coin du feu - ou censés l'avoir été. Seul nous retiendra le premier livre, paru en 1584, qui consacre sa troisième section à traiter «Des Femmes, et des Filles ». L'anecdote catalane est mise ici dans la bouche d'une femme qui dit la tenir de son mari, lequel allègue lui-même « un Docteur en Droict » bientôt désigné comme Bohier ${ }^{24}$. Mais on relève d'intéressantes variations : au lieu que le mari honore sa femme dix fois qualibet die (" par chacun jour », traduisait Du Verdier), il consent à présent des exceptions pour se conformer aux interdits de l'Église («si ce n'estoit les jours que le Calendrier estoit marqué de rouge », ibid.) ; et un autre exemple de dérèglement conjugal nous est allégué, celui de Polémon (non plus le philosophe platonicien, mais le roi du Pont) envers sa femme Veronica, en qui il faut reconnaitre Bérénice, la future maîtresse de Titus, - encore que Flavius Josèphe, dans la traduction Génébrard des Antiquités judaïques, n'attribue pas tant la répudiation aux assauts importuns du mari

${ }^{22}$ Histoire des plantes, IX, XVIII, 9 ; Histoire naturelle, XXVI, LXIII, 99 ; Deipnosophistes, I, 32. Cf. Agrippa, De incertitudine, ch. 63 (trad. Turquet de Mayerne, [Genève], Jean Durand, 1582, p. 273-274); et Rabelais, Tiers livre, ch. XXvII (éd. Huchon, p. 437).

${ }^{23}$ Voir G.-A. Pérouse, Nouvelles françaises du XVI siècle, Genève, Droz, 1977, ch. XVI, pp. 342-344.

${ }^{24}$ Éd. C.E. Roybet, Paris, Lemerre, 1873-1882, t. I, livre I, 3e Serée, « Des Femmes, et des Filles », p. 103. 
qu'à « l'intempérance de Bernice [sic], qui le laissa $»^{25}$. Le récit est surtout pris en charge par plusieurs narrateurs, qui n'entendent pas en tirer la même leçon ni le même profit, et c'est la raison pour laquelle il s'interrompt en plein milieu de l'histoire, avant même l'arrêt rendu par la reine d'Aragon. Le mari s'est en effet bien gardé d'en révéler le contenu à sa femme, pour n'être pas lui-même réfréné dans ses ardeurs sexuelles (dix fois valent mieux que six !). Mais courait-il vraiment grand risque, alors qu'on nous répète à deux reprises, en écho à Du Verdier, que « les femmes se plaignent souvent de peu, mais non pas de trop »(p. 103 et 104) ? Toujours est-il que le fin mot de l'histoire ne sera livré qu'aux hommes, et encore « à l'oreille» (p. 104), l'auditoire féminin étant laissé dans l'ignorance de l'arbitrage rendu en sa faveur.

Du moins dans l'édition posthume de 1608 (reproduite par Roybet), qui étoffe sensiblement le texte de 1584 , celui que Montaigne a pu éventuellement connaître ${ }^{26}$. Sous sa forme primitive il est en effet deux fois moins long. L'anecdote proprement dite s'y présente sous un jour un peu différent, puisque silence est encore fait sur l'aveu à la reine des dix rapports conjugaux quotidiens et que l'époux poitevin s'abstient de répéter, après l'interlocuteur précédent, la glose misogyne de Bohier, accompagnée de ses références savantes au Code de Justinien (« selon la loy Quisquis, [...] ce dit Lucas de Penna », p. 104). Mais les deux versions enchaînent bien l'une et l'autre sur les «petits registres » du villageois ennemi des femmes et sur les bons mots qui y sont consignés («Faites-le si souvent, qu'il ne vous revienne pas à un liard. [...] Faites-le donc encor un coup pour le demeurant de vostre argent », p. 105).

Quelques mois plus tard, paraissent Les Matinées du seigneur de Cholières, dont la neuvième et dernière s'intitule « De la trefve conjugale $»^{27}$. Un brave paysan, Dominique, s'y plaint à un homme d'Église, le bien-nommé Théodat, que sa femme se refuse à lui et il se voit bientôt expliquer la nécessité de certaines périodes d'abstinence. Le religieux commence par alléguer S. Augustin (à qui se référait aussi l'époux poitevin mis en scène par Bouche ${ }^{28}$ ), mais au lieu de distinguer entre intempérance conjugale et paillardise, fût-ce pour les condamner toutes les deux, il les assimile purement et simplement l'une à l'autre : « les maris ne laissent point de

${ }^{25}$ Livre XX, ch. v (Paris, Toussaint du Bray, 1609, t. I, p. 722). Pour une autre version des faits, incriminant Polémon propter nimietatem coitus, voir le Dictionnaire historique et critique de Bayle (Rotterdam, 1702), s.v. Bérénice, n. A $\Delta$, t. I, pp. 566-567.

${ }^{26}$ Sur Montaigne lecteur de Bouchet, voir P. Villey, Les Sources et l'évolution des Essais de

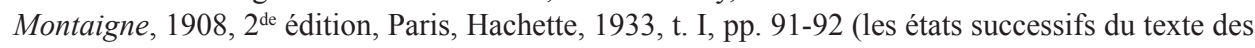
premières Serées n'y semblent toutefois pas pris en compte) ; et sur Bouchet lui-même lecteur du «Seigneur de Montagne », voir A. Janier, Les Serées du libraire-imprimeur Guillaume Bouchet, Paris, Champion, 2006, $2^{\mathrm{e}}$ partie, ch. VII, pp. 541-551.

27 Euvres, éd. É. Tricotel, Paris, Librairie des Bibliophiles, 1879, t. I, pp. 299-325.

${ }^{28}$ Du moins dans le texte de 1608 (« luy remonstrant [au mari catalan] ceste Roine ce que sainct Augustin a delaissé par escrit : que l'excez en la conjonction maritale estoit aussi bien à reprendre que la paillardise », p. 104). 
paillarder, lesquels à toutes heurtes [à chaque instant, continuellement] sur-saillissent leurs femmes ». Et il enchaîne assez abruptement sur le cas tiré des Decisiones Burdegalenses:

un homme de Cataloigne [...] sur-chargeoit si souvent sa femme qu'elle fut contrainte (rara auis in terris, alboque simillima coruo) en faire sa plainte à la royne d'Arragon, laquelle aiant fait appeler le mary, confessa que veritablement dix fois par jour ils joüoient par ensemble à la beste à deux dos. Dont la royne fut fort esbahie [...]» (p. 307).

L'affaire est rapportée sans déférence excessive pour l'éminent jurisconsulte. Le terme sur-chargeoit fait plaisamment écho au sur-saillissent de la phrase précédente, tandis que jouer à la beste à deux dos introduit dans le discours du clerc une savoureuse note rabelaisienne ${ }^{29}$. S'agit-il de se mettre à la portée de son interlocuteur? ou de se gagner la faveur du public, en mettant les rieurs de son côté ? Le sel de la plaisanterie se trouve dans la parenthèse ironique rara auis in terris, alboque simillima coruo [un oiseau aussi rare qu'un merle blanc], variante du vers de Juvénal rara auis in terris, nigroque simillima cycno [aussi rare qu'un cygne noir] (Sat., VI, 165) ${ }^{30}$, qui se rapporte à une femme pourvue de toutes les qualités, c'est-à-dire introuvable, mais ici, dans le même contexte misogyne, ainsi faite qu'elle puisse se plaindre d'être trop besognée plutôt que pas assez. Et c'est au compte de la reine d'Aragon qu'est mis ensuite un piquant rapprochement avec le treizième travail d'Hercule, le dépucelage de toutes les filles de Thespios, suivant les versions en cinquante nuits, en sept, voire en une seule. Plaisant contraste avec notre Dominique, qui se contente, dans le meilleur des cas, de deux rapports sexuels mais dont le début de la réplique, dirigé contre « ceux lesquels sans aucune discretion s'enyvrent de leur vin, et se jettent sur leur viande, comme un pourceau sur les raves » (pp. 307-308), trahit l'emprunt à Du Verdier, tout comme, dans la bouche de Théodat, la référence précise à Bohier et à « ses decisions du Parlement de Bordeaux » (p. 307).

Un mot, enfin, sur Brantôme, le voisin périgourdin de Montaigne, qui se met lui aussi à composer dans ces années-là, même si, comme on le sait, son Recueil des dames ne paraîtra que bien plus tard, au siècle suivant. Dans le chapitre que le second livre (devenu pour la postérité Les dames galantes) fait porter « [s]ur les femmes mariés, les veuves et les filles », il insère, entre deux historiettes mettant en scène de lascives héroïnes, l'histoire de notre Catalane, qui,

un jour que le grand Alfonce, Roy d'Arragon, faisoit son entrée dans Sarragosse, se vint jetter à genoux devant luy et luy demander justice. Le Roy, ainsi qu'il la vouloit ouir, elle demanda

${ }^{29}$ Garg., ch. 3 (éd. Huchon, p. 15). Métaphore usuelle dans les textes des XIV et XV siècles suivant L. Sainéan, La langue de Rabelais, Paris, De Boccard, 1922-1923, t. II, p. 301 (qui renvoie curieusement aux chapitres 21 et 23 du premier roman).

${ }^{30}$ Par contamination avec VII, 202, «Felix ille tamen coruo quoque rarior albo ». Coruus albus forme l'adage $\mathrm{n}^{\circ} 3635$ d'Érasme, qu'on retrouve dans le colloque Uxor mempsigamos (« Les maris de cette sorte sont aussi rares que les corbeaux blancs », trad. J.-Cl. Margolin, éd. citée, p. 284). 
luy parler à part, ce qu'il luy octroya : et, s'estant plaincte de son mary, qui couchoit avec elle trente deux fois tant de jour que de nuict, qu'il ne lui donnoit patience, ny cesse ny repos, le Roy, ayant envoyé querir le mary et sceu qu'il estoit vray, ne pensant point faillir puisqu'elle estoit sa femme, le Conseil de Sa Majesté assemblé sur ce fait, le Roy arresta et ordonna qu'il ne la toucheroit que six fois ${ }^{31}$.

La singularité du cas a été d'emblée soulignée par la formule d'ouverture du récit ( « Ces Dames ne ressembloient pas une Dame espaignolle... »), que vient relayer l'attaque de l'anecdote suivante (« À ceste Dame n'estoit pas ressemblante une fille, Damoiselle de maison... »). Brantôme enchaîne en effet sur l'histoire d'une jeune mariée qui vient se plaindre, le lendemain de ses noces, de n'avoir été honorée de son nouvel époux que quatre fois (et encore avec de longs intervalles), au lieu de la douzaine escomptée, ce qui permet au narrateur de conclure que « tout le monde ne ressemble pas le Gentilhomme espaignol » du conte précédent (p. 472). Car le contexte et le cadre social ont bien changé : le récit est détaché, non plus d'un recueil de décisions judiciaires, mais d'une chronique historique qui met en scène « le grand Alfonce, Roy d'Arragon », c'est-à-dire Alphonse V, qui, en souverain justicier, écoute la plaignante, convoque la partie adverse et, après avis de son conseil, rend son arrêt. Le commentaire final du monarque, ciselé autour d'une vigoureuse antithèse entre la " grande challeur et puissance » du mari et la « grande froideur et continance » de l'épouse, aussi singulières les unes que les autres, aurait pu être enregistré par Panormita [Antonio Beccadelli] dans les De dictis et factis Alphonsi Regis Aragonum libri quatuor, mais nous ne l'y avons pas retrouvé, contrairement à d'autres dits notables allégués par Montaigne dans les Essais, soit explicitement (« Le Roy Alphonse disoit que les asnes estoyent [...] de meilleure condition que les Roys : leurs maistres les laissent paistre à leur aise, là où les Roys ne peuvent pas obtenir cela de leurs serviteurs », I, 42, p. 287) ${ }^{32}$, soit de manière plus allusive, précisément dans ce même chapitre « Sur des vers de Virgile » (« Celuy là s'y entendoit, ce me semble, qui dit qu'un bon mariage se dressoit d'une femme aveugle, avec un mary sourd », III, 5, p. 914) ${ }^{33}$. Malgré l'habillage courtisan et princier dont Brantôme a revêtu notre vieille histoire, sa source pourrait bien être à nouveau les Decisiones Burdegalenses de Bohier, lues cette fois dans leurs premières éditions, celles où nous avons rencontré un roi d'Aragon, non

${ }^{31}$ Éd. Vaucheret, p. 471 (l'apparat critique ne fait pas le rapprochement avec Montaigne).

32 «Asinorum conditionem longe meliorem quam regum, illis quidem comedentibus dominos parcere, regibus neminem ", livre I, ch. 13 (Bâle, Hervagianus, 1538, p. 4). Un bon mot que les index des éditions modernes, après Villey, réfèrent à tort à Alphonse XI de Castille (« La Pochothèque », p. 1810 ; « La Pléiade », pp. 1926-1927).

33 "Matrimonium ita demum exigi tranquille, et sine querela posse dicebat, si mulier cæca fiat, et maritus surdus » (livre III, ch. 7, p. 71 - repris par Érasme dans ses Apophthegmata, VIII, IV). Sur la réception des Dicta et facta Alphonsi Regis Aragonum à la Renaissance, voir B. Bowen, « Rabelais and the Rhetorical Joke Tradition », in Rabelais 's Incomparable Book, éd. R. La Charité, Lexington, French Forum, 1986, pp. 213-225, ici p. 218. 
une reine, et un mari uxorem suam XXX. vicibus cognoscentem, ce qui correspond, à peu de chose près, aux trente-deux rapports sexuels mentionnés ici.

En va-t-il différemment de Montaigne ? On a peine à imaginer que l'ancien magistrat du Parlement de Bordeaux, membre pendant dix ans de sa Chambre des Enquêtes ${ }^{34}$, n'ait pas assidûment consulté et pratiqué le recueil jurisprudentiel d'un ancien Président de la cour. Comme le fait observer André Tournon, il donne de l'anecdote une version très proche de celle de Bohier et signe même pratiquement son emprunt en notant, immédiatement après, la réaction indignée des « docteurs » ${ }^{35}$. Mais l'essentiel n'est pas là. Il réside plutôt dans l'utilisation tout à fait singulière que Montaigne fait de ce litige conjugal et dans la manière dont il l'insère dans un développement plus vaste, au moyen du double connecteur Et que (à relier, en amont, à « Après que nous avons cogneu... » et à " Et en outre, que nous avons appris... », p. 896 ; en aval, à « Après avoir creu (dis-je) et presché cela », p. 897, anaphore à valeur résomptive, ou récapitulative). Il y a là, comme le notent à juste titre les éditeurs de "La Pléiade », une parodie de style judiciaire, qui vise à reproduire les divers attendus d'un jugement et à imiter « la formulation des arrêts syntaxiquement très complexe et très lourde $\|^{36}$. Regardons-y d'un peu plus près, en remontant jusqu'à l'étonnante prise de position féministe qui ouvre ce développement (« Les femmes n'ont pas tort du tout, quand elles refusent les reigles de vie, qui sont introduites au monde : d'autant que ce sont les hommes qui les ont faictes sans elles », p. 896) et qui sera reprise, près de quatre siècles plus tard, par Simone de Beauvoir dans l'Introduction du Deuxième sexe. D'où nos désaccords incessants, à défaut d'une entente qui ne saurait être, dans le meilleur des cas, qu'orageuse et tumultueuse. Suit une référence sibylline à un auteur déjà allégué (Platon ou Virgile, suivant les rares éditeurs modernes qui se risquent à insérer ici une note ${ }^{37}$ ), laquelle annonce le début du raisonnement au moyen du démonstratif à valeur cataphorique cecy. Point de départ : la plus grande ardeur amoureuse des femmes que des hommes, attestée par la fable de Tirésias (avec citation d'Ovide tirée des Métamorphoses, III, 323) ${ }^{38}$, puis par les exemples de Proculus et de Messaline (" seulement» dix rapports sexuels en une nuit, contre vingt-cinq !), et enfin par le cas catalan, où l'intérêt se porte davantage sur la reine que sur l'épouse. Celle-ci inspire bien à Montaigne un long commentaire, qui ajoute au caractère étonnant et

${ }^{34}$ Sur Montaigne au Parlement de Bordeaux et, plus précisément, à la Chambre des Enquêtes, voir A. Tournon, Montaigne en toutes lettres, Paris, Bordas, 1989, pp. 19-22; et Montaigne. La glose et l'essai, op. cit., pp. 185-202.

${ }_{35}$ La glose et l'essai, ch. IV, n. 50, p. 376.

${ }^{36}$ P. 1743 (n. 5 sur p. 897).

37 Platon, La République, V, 451c sqq. suivant les éditeurs de «La Pléiade », Virgile (sans doute pour l'Énéide, VIII, 387-392 et 404-406, cité supra p. 891) suivant G. de Pernon, auteur d'une traduction en ligne et sur papier des Essais (Léogeats, Pernon-Éditions, 2009, 3 vol.).

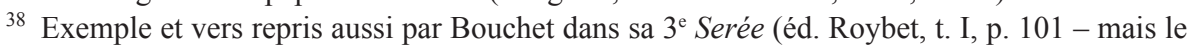
vers ne sera introduit que dans la réédition de 1608). 
à peine croyable de cette plainte l'idée d'une mise en cause dévoyée de l'autorité maritale, mais le propos est rejeté à l'intérieur d'une parenthèse. Tel n'est pas le cas de l'appréciation portée ensuite sur l'arrêt rendu, par lequel cette «bonne reine », en fixant la limite à six fois par jour, ne semble pas tant s'être souciée de modérer les ardeurs d'un mari en particulier que de réfréner les appétits désordonnés des femmes (« [r]elaschant et quitant beaucoup du besoing et desir de son sexe », p. 897). Ce qui ne fait somme toute que confirmer les juristes, Tiraqueau, Bohier et consorts, dans leurs préjugés misogynes. Le segment textuel qui suit ${ }^{39}$ ne figurait pas dans les éditions parues du vivant de Montaigne. Cet ajout vient renforcer l'opposition entre la libido jamais assouvie des femmes et celle, plus réglée, des hommes (désignée par " nos appétits », ibid.), que le législateur athénien Solon, suivant Plutarque ${ }^{40}$, jugeait possible d'abaisser à trois rapports par mois sans contrevenir aux lois du mariage. Au terme de ce long et tortueux raisonnement, que Montaigne lui-même juge nécessaire de résumer pour ne pas complètement égarer son lecteur («Apres avoir creu (dis-je) et presché cela »), il énonce enfin ce qu'il avait annoncé une trentaine de lignes plus haut, à savoir en quoi nous « traictons inconsiderément» les femmes. Alors qu'elles sont indiscutablement, par nature, plus concupiscentes que nous, nous leur imposons une continence et une chasteté auxquelles nous ne nous plions pas nous-mêmes et serions bien incapables de nous soumettre. Nous leur demandons tout et son contraire, d'être « saines, vigoreuses, en bon point, bien nourries, et chastes ensemble : c'est-à-dire, et chaudes et froides » (p. 898). Cette antithèse déjà rencontrée sous la plume de Brantôme se rapporte ici uniquement aux femmes dont, une fois de plus, et contrairement à tant de ses contemporains, Montaigne prend la défense. Nous retrouvons l'entrée de l'Index dont nous étions partis, « Elles [les femmes] n'ont tort de refuser une trop grande continence $»$.

Concluons rapidement sur le mariage selon Montaigne, non sans rendre hommage, en terre polonaise, aux travaux que Kazimierz Kupisz, de l'Université de Łódźt a naguère consacrés au sujet, et avec, comme lui, le souci de souligner la cohérence du discours tenu d'un bout à l'autre des Essais. Deux citations pourront y suffire. La première sera tirée du chapitre " De la moderation », où Montaigne consacre un long développement à l'union conjugale : «C'est une religieuse liaison et devote que le mariage : voylà pourquoi le plaisir qu'on en tire, ce doit estre un plaisir retenu, serieux et meslé à quelque severité : ce doit estre une volupté aucunement prudente et conscientieuse » (I, 29/30, pp. 204-205). La seconde nous ramènera à « Sur des vers

${ }^{39}$ Depuis « considerans le divers jugement... » jusqu'à « cette hantise conjugale » (ibid.).

40 De l'amour, XXIII, 769A, trad. Amyot de 1572, f. 612C. Cf. Vie de Solon, 20, 4 (même traducteur, éd. Walter, Paris, Gallimard, 1951, t. I, p. 195). Voir aussi Bouchet, Serées 22 et 26, éd. Roybet, t. III, p. 294, et IV, p. 163.

${ }^{41}$ Voir Elle et lui. Problématique féminine des Essais [Folia litteraria, 12, 1985], en particulier ch. IV « Le mariage ». 
de Virgile » : «Il faut (dit Aristote ${ }^{42}$ ) toucher sa femme prudemment et severement, de peur qu'en la chatouillant trop lascivement, le plaisir ne la face sortir des gons de la raison » (III, 5, pp. 891-892 $)^{43}$. On ne saurait donc trop conseiller à un jeune homme de ne point s'unir trop tôt à une épouse... et Montaigne lui-même eut la sagesse, La Boétie disparu, d'attendre, pour se (laisser) marier, l'âge de trente-deux ans !

\section{BIBLIOGRAPHIE}

Bohier, N. (1547). Decisiones Burdegalenses. Lyon : Antoine Vincent. Brantôme (1991). Recueil des dames. C.E. Roybet (éd.), Paris : Lemerre.

Cholières (1879). Les Matinées, t. 1, É. Tricotel (éd.), Librairies des Bibliophiles.

Henry, P. (1977, oct.-déc.). Les titres façades, la censure et l'écriture défensive chez Montaigne.

Bulletin de la Société des Amis de Montaigne, 24 (5), 11-28.

Janier, A. (2006). Les Serées du libraire-imprimeur Guillaume Bouchet. Paris : Champion.

Kupisz, K. (1985). Elle et lui. Problématique féminine des Essais, t. 12. Folia litteraria.

Montaigne, M. (2007). Les Essais, B.J.-S.C. (éd.), Paris : Gallimard.

Pérouse, G.-A. (1977). Nouvelles françaies $d u X V I^{e}$ siècle. Genève : Droz.

Rabelais, F. (1994). Euvres complètes, H.M. (éd.), Paris : Gallimard.

Tournon, A. (2000). Montaigne. La glose et l'essai, $2^{\mathrm{e}}$ édition. Paris : Champion.

Verdier du, A. (1580). Les Diverses Leçons. Lyon: Étienne Michel.

Villey, P. (1933). Les sources et l'évolution des Essais de Montaigne. Pari : Hachette.

${ }^{42}$ Histoire des animaux, X, v, 636b.

${ }^{43}$ Répété par Bouchet, 23e Serée, t. IV, p. 54. 